\title{
In pursuit of brevity?
}

Our obsession with making everything shorter and easier may fit our ever-increasing overload of both information and activity, but does it deliver?

Mike Grace m.grace.bdj@bda-dentistry.org.uk
W

e live in a world of the 'sound bite', the 'summary' and the 'abridged version'. Film distributors force directors to release films cut back to a certain time length, television series split into short programmes of $\mathbf{5 0}$ minutes maximum and books are now being published as 'short books' cut back to 20,000 words. Even the BDJ now provides 'In Brief' boxes and summary pages for all the articles and the research papers in an attempt to provide the essence of each paper in a shortened easyto-read format to enable people to save time.

At first sight this approach is very attractive, especially to the busy practitioner. But do we really get the value we could if we adopt this 'short-term' approach? Our obsession with making everything shorter and easier may fit our everincreasing overload of both information and activity, but does it deliver? In fact, are we fooling ourselves and our pursuit for brevity is actually making life more difficult? More significantly, are we letting this cultural phenomenon deaden the level of quality we deliver, especially in the area of treatment planning?

In this issue of the $B D J$ we are publishing an interesting paper in the Education Section (page 152) which considers a teaching aid (a CAL programme) that helps students understand how to plan clinical care more effectively, and discusses the difference between planning and doing. People often focus more on 'doing' things than 'thinking about what to do', because they believe there just is not enough time to do all they want to do? This is the cliché between being efficient and being effective. Being efficient means doing things in the right way (so the cliche goes) but being effective means doing the right thing. In our pursuit for speed and brevity we are actually making it harder to decide what the right thing is.

What the paper does not address is how to teach the skills required to think and plan, which is hardly surprising as very few papers and articles do address this aspect of treatment planning. The reason for this may be because it is a common misconception that people are already knowledgable and skilled in thinking and planning and so text books, lecturers and courses on treatment planning move straight to the clinical aspects. Another factor is there is so little time available in the syllabus. However, we must remember that until recently the same happened with communication skills, where the ability to communicate effectively was assumed rather than taught until the subject entered the curriculum for dental students. How long will it be, I wonder, before the same happens to the skills of thinking and planning?

Quality care for patients must always be the overiding motivation for what we do, and as such I welcome a paper drawing attention to a method for helping teach understanding in planning. In the past too much has been left to chance when it comes to teaching this important skill (in both undergraduate and postgraduate programmes) leaving individuals to develop their own abilities as best they can. This is even more important as society continues to encourage the 'short term' culture we see developing around us which lulls us into believing that we have so little time.

To cope with our need for ever-increasing activity in ever-diminishing time we reduce the opportunities to think, to reflect and to formulate, and as a result we plan less. Because we think and plan less, we lose the ability to be as effective, fooling ourselves into believing that planning is not that important because we cannot predict the future with any certainty any more. There is a danger that in time this will affect everything we do, including the provision of patient care.

Let us always remember - we all have 24 hours a day. It is how we choose to use it that matters. 